\title{
Low-Cost Far-Infrared FPA based on High-Volume Pressure Sensor Process
}

\author{
Michael Krueger ${ }^{1}$, Ingo Herrmann ${ }^{2}$ \\ ${ }^{1}$ Robert Bosch GmbH - Automotive Electronics, Tuebinger Str. 123, D-72762 Reutlingen, Germany, \\ michael.krueger@de.bosch.com \\ ${ }^{2}$ Robert Bosch GmbH - Corporate Research, Robert-Bosch-Platz 1, D-70839 Gerlingen, Germany
}

\begin{abstract}
In order to achieve substantial cost reduction for far-infrared focal plane arrays (FPA), consequent adoption of existing high-volume MEMS processes is required. For that reason we used a high-volume pressure sensor process for fabrication of thermodiode FPA. While $1^{\text {st }}$ Gen FPA successfully proved the suitability of the fabrication process, performance was strongly improved for the $2^{\text {nd }}$ Gen FPA to meet customer demands for low-cost thermal imaging and thermal sensitivity of 270 mK @ f/1.0 and $9 \mathrm{~Hz}$ frame rate was achieved. In addition development of the $3^{\text {rd }}$ Gen FPA with QVGA format, $28 \mu \mathrm{m}$ pixel pitch and $100 \mathrm{mK}$ thermal sensitivity is ongoing.
\end{abstract}

Key words: Focal plane array, low-cost far-infrared imaging, thermodiode, APSM process

\section{Introduction}

Far-infrared imaging with uncooled focal plane arrays enables a widespread field of applications in different market segments like automotive, industry and security. Nevertheless the production volumes of FPAs are still very limited due to a high price level very unusual for MEMS markets. Key point for establishing a real high-volume market is the availability of FPA and infrared optics for a strongly reduced price level. For many applications even a performance reduction compared to wellestablished high-end FPA is acceptable.

Typically resistive FPA made from $\mathrm{VOx}$, a-Si or SiGe, but also thermodiode FPA use dedicated processes for this kind of device [1,2]. However dedicated processes in conjunction with low production volumes are a fundamental barrier for significant cost reduction as known from high-volume MEMS products like pressure sensors, accelerometers or gyroscopes.

The approach shown in this paper is the other way round: in order to produce low-cost FPA we use a high-volume MEMS process for pressure sensors. This process is in production since 2007 and more than 100 million sensors were fabricated since then. By this means substantial economies-of-scale become possible for low-cost FPA production.

\section{Low-cost thermodiode FPA concept}

The high-volume fabrication process of the thermodiode FPA is based upon the unique Advanced Porous Silicon Membrane (APSM) process [3] which enables the fabrication of free-standing membranes made of monocrystalline silicon. This material allows the formation of pn junctions with a very low $1 / \mathrm{f}$ noise. The combination of low-cost micromechanics with excellent electrical performance offers the opportunity for fabrication of thermally isolated pn junctions, also called thermodiodes [2]. Compared to resistive bolometers the thermodiodes also offer the advantage that the sensitivity of the sensing element does not depend on process variations of the resistive layer or the MEMS process, but only on the very well defined semiconductor processes for fabrication of pn junctions. Moreover the sensitivity is less sensitive on device temperature compared to resistive bolometers. Last but not least the selfdecoupling capability of diode arrays enables addressing from the array sides without the need of switching elements underneath the pixels. 


\section{Generations of thermodiode FPA}

Work on thermodiode FPA at Bosch started in 2007 with the public funded project ADOSE (Tab. 1). The $1^{\text {st }}$ Gen of FPA successfully demonstrated the suitability of the APSM process for thermodiode fabrication. The device consisted of a $42^{*} 28$ pixel array with a relatively large pixel pitch of $230 \mu \mathrm{m}$ and four thermodiodes per pixel. The Read-out IC (ROIC) part was monolithically integrated sideby-side to the pixel array (Fig. 1).

Tab. 1: Generations of thermodiodes FPA

\begin{tabular}{|c|c|c|c|}
\hline & $1^{\text {st }}$ Gen & $2^{\text {nd }}$ Gen & $3^{\text {rd }}$ Gen \\
\hline Status & $\begin{array}{c}\text { Study } \\
\text { finished } \\
\text { (ADOSE) }\end{array}$ & $\begin{array}{c}\text { Series } \\
\text { development } \\
\text { ongoing }\end{array}$ & $\begin{array}{l}\text { Study ongoing } \\
\text { (RTFIR) }\end{array}$ \\
\hline $\begin{array}{l}\text { Pixel } \\
\text { Count }\end{array}$ & $42 * 28$ & $\begin{array}{l}100 * 50 \\
80 * 60\end{array}$ & $320 * 240$ \\
\hline $\begin{array}{l}\text { Pixel } \\
\text { Pitch }\end{array}$ & $230 \mu \mathrm{m}$ & $100 \mu \mathrm{m}$ & $28 \mu \mathrm{m}$ \\
\hline $\begin{array}{l}\text { NETD } \\
@ \text { f/1.0 }\end{array}$ & $\begin{array}{l}400 \mathrm{mK} \\
@ 3 \mathrm{~Hz}\end{array}$ & $\begin{array}{c}270 \mathrm{mK} \\
@ 9 \mathrm{~Hz}\end{array}$ & $\begin{array}{c}100 \mathrm{mK} \\
\text { (Specification) }\end{array}$ \\
\hline
\end{tabular}

Based on these successful results the $2^{\text {nd }}$ Gen FPA was designed to meet more demanding targets for pixel count, pixel pitch, frame rate and thermal sensitivity. The concept of the device remained identical to the $1^{\text {st }}$ Gen except for poly silicon replacing AISiCu as electric lead material for the suspensions in order to enhance thermal isolation. Based on positive customer feedback series development has been started for the commercialization of the $2^{\text {nd }}$ Gen FPA.

The sensor concept shown in Fig. 1 allows the use of well-established design and fabrication processes at Bosch. However, pixel pitch and by this means the number of pixels are limited due to common layout rules for both ROIC and thermodiode pixels. In order to broaden the portfolio, development of the $3^{\text {rd }}$ Gen FPA was started with the support of the public funded project RTFIR.

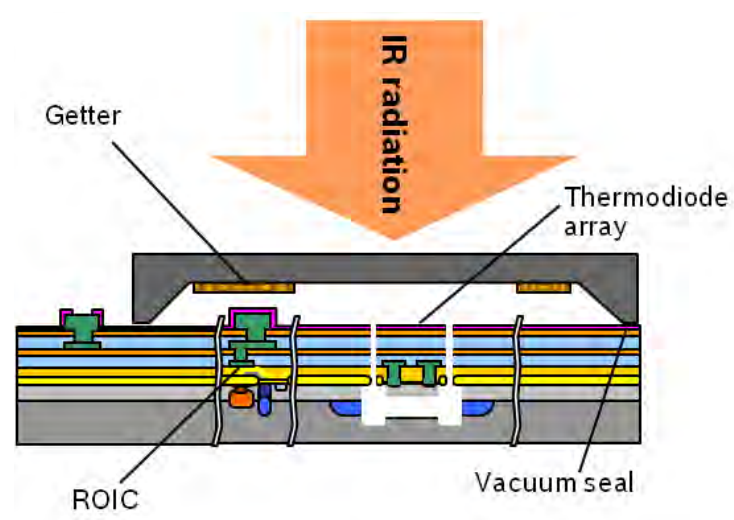

Fig. 1. Schematic of $1^{\text {st }}$ and $2^{\text {nd }}$ Gen FPA with ROIC side-by-side to the pixel array. Glasfrit waferbonding and getter provide vacuum inside the cavity.

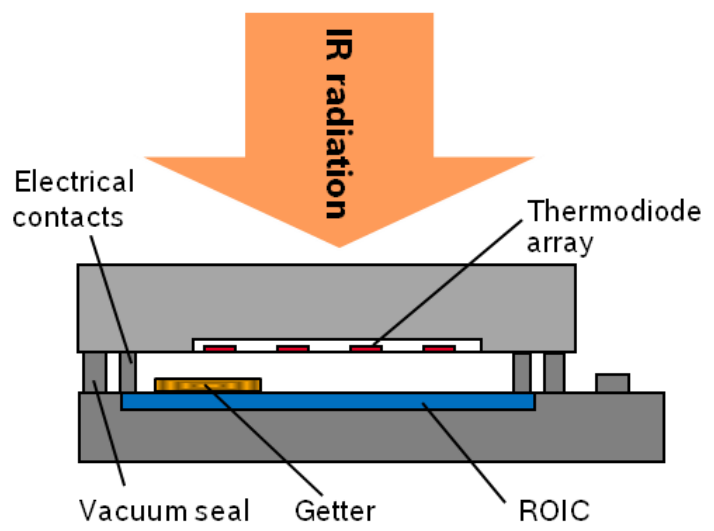

Fig.2: Schematic of 3rd Gen FPA.

The $3^{\text {rd }}$ Gen will continue to use thermodiodes using the APSM process. The ROIC will make use of an advanced CMOS process and will be attached to the sensor by a waferbonding process (Fig. 2). By this means thermodiodes and ROIC can be optimized separately. The waferbonding process will provide both vacuum encapsulation and electrical contacts from sensor chip to ROIC. This unique concept is enabled by the self-decoupling property of a diode array. While resistive bolometer pixels require some type of switching element per pixel, a diode array can be accessed using just row and column lines, reducing the number of required contacts to the ASIC by more than 2 orders of magnitude. 


\section{Sensor process flow and sensor design}

The fabrication of the thermodiode FPA starts with the so called Advanced Porous Silicon Membrane (APSM) process. The main steps of this surface micromachining process are local anodic etching of porous silicon, followed by thermal rearrangement of the porous silicon and finally epitaxial growth of a silicon layer. More details on the process can be found in [3]. By this means a monocrystalline membrane layer on top of vacuum cavities can be formed in the region of the pixel array.

Following the APSM process, pn junctions are implanted in the monocrystalline silicon membrane. As a next step, dielectric and metallization layers are deposited (Fig. 3), using the standard high-volume process for pressure sensor fabrication. For $1^{\text {st }}$ Gen and $2^{\text {nd }}$ Gen FPA these process steps are also used for ROIC fabrication side-by-side to the pixel array. Then the pixels are released by etching the dielectric layers and the monocrystalline silicon membrane. Afterwards isotropic undercutting is applied to remove the monocrystalline silicon underneath the suspensions in order to reduce their thermal conductivity.

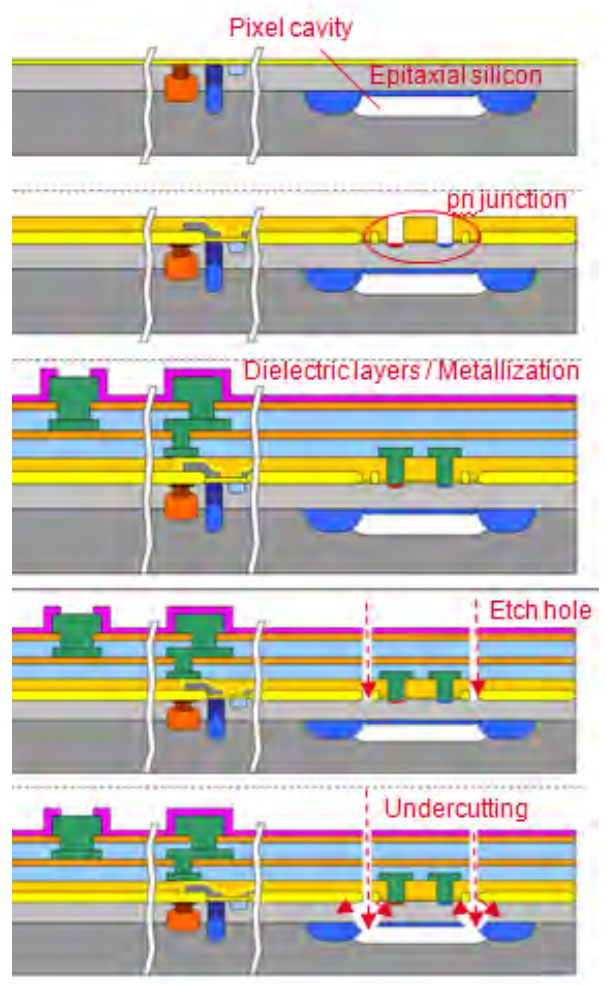

Fig. 3. Process flow for fabrication of the pn junctions and the ROIC and for release of the pixels.
Fig. 4 and 5 show the chip layout for $1^{\text {st }}$ and $2^{\text {nd }}$ Gen FPA. Additionally a single pixel with four pn junctions in series and the suspensions for thermal isolation and electrical interconnects is shown in Fig. 4. Pixel pitch and fill factor are $230 \mu \mathrm{m}$ and $56 \%$ for $1^{\text {st }}$ Gen FPA and $100 \mu \mathrm{m}$ and $52 \%$ for $2^{\text {nd }}$ Gen FPA respectively.

For $1^{\text {st }}$ and $2^{\text {nd }}$ Gen FPA wafer processing is completed by standard glasfrit waferbonding to provide vacuum encapsulation. The getter is located on the inner surface of the silicon cap wafer.

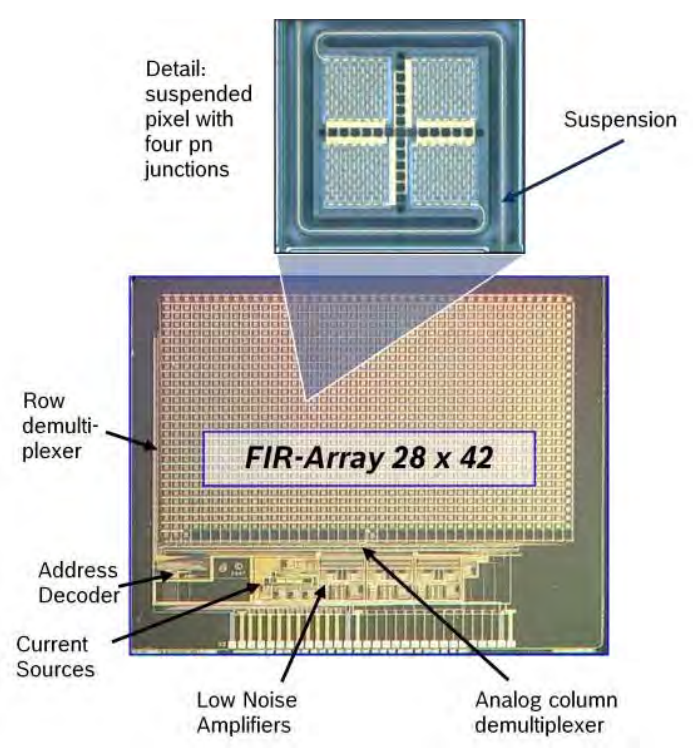

Fig. 4. Picture of $1^{\text {st }}$ Gen FPA and single pixel with 4 thermodiodes (without cap wafer).

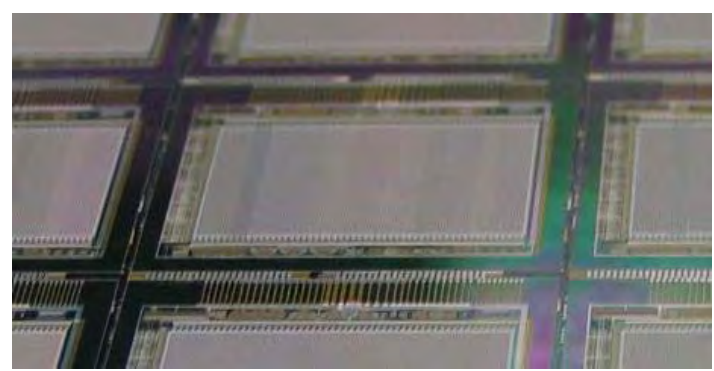

Fig. 5. Picture of $2^{\text {nd }}$ Gen FPA with $100 * 50$ pixel on wafer (without cap wafer).

For $3^{\text {rd }}$ Gen FPA the sensor chip will no longer contain the ROIC. As a result, the process flow and layout rules can be adapted to decrease the pixel pitch, thermal mass and sensitivity. 


\begin{abstract}
Results
$1^{\text {st }}$ Gen FPA was designed as proof-of-concept for thermodiodes using the APSM process. With relatively large pixels $(230 \mu \mathrm{m}$ pitch), a low pixel count of $42^{*} 28$ pixels and a 3 -channel differential amplifier a thermal sensitivity (Noise equivalent temperature difference - NETD) of $400 \mathrm{mK}$ was achieved at a frame rate of $3 \mathrm{~Hz}$ and with $f / 1.0$ optics. Detailed noise analysis revealed that the ROIC was the dominating noise source. Using an improved low noise amplifier, NETD of $<50 \mathrm{mK}$ seems feasible as a result of the advantageous large absorption area of the pixels.
\end{abstract}

For $2^{\text {nd }}$ Gen FPA the requirements were adapted to achieve a competitive specification regarding pixel count and pixel pitch, thermal sensitivity (NETD) and frame rate. In addition a low power consumption of only $25 \mathrm{~mW}$ was achieved.

With $100 * 50$ pixels and $100 \mu \mathrm{m}$ pixel pitch a thermal sensitivity of $180 \mathrm{mK} @ \mathrm{f} / 0.8$ and $9 \mathrm{~Hz}$ frame rate was achieved, corresponding to 270mK @ f/1.0. The NETD measurement was performed at room temperature after calibration at $25^{\circ} \mathrm{C}$ and $35^{\circ} \mathrm{C}$ blackbody temperature.

Frame rate for $2^{\text {nd }}$ Gen FPA is limited by the thermal time constant of heat flow from pixel to substrate. Measurements of the thermal time constant were performed by exploiting the selfheating effect of the pixels: the electrical current through the pixel was changed and the time dependency of the applied voltage was analyzed. This resulted in a thermal time constant $\mathrm{t}_{63 \%} \approx 120 \mathrm{~ms}$ which is sufficient for 9 $\mathrm{Hz}$ frame rate, well-suited for many applications and fitting to export control regulations.

In order to quantify the heat dissipation through the suspensions and the residual gas, the thermal time constant was also measured at different pressure values inside a vacuum chamber using a sensor chip without cap. The measurements confirmed that for time constants $t_{63 \%} \approx 120 \mathrm{~ms}$ the heat dissipation through the suspensions exceeds the one through the residual gas.

This demonstrates how effective the getter reduces the residual gas pressure taking into account outgasing during standard glasfrit bonding.

In addition, the quality of the thermodiodes was characterized using their thermal coefficient. Typical values of $-2,1 . .-2,2 \mathrm{mV} / \mathrm{K}$ were measured per pn junction showing good agreement with theoretical calculations [4].
A typical image taken with these $2^{\text {nd }}$ Gen FPA is shown in Fig.6.

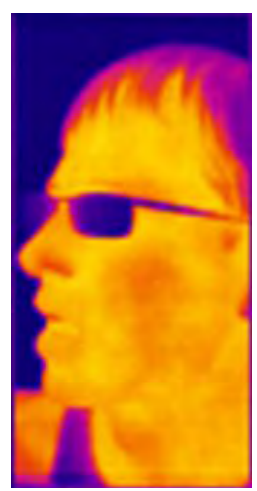

Fig. 6. Example of image taken with $2^{\text {nd }}$ Gen FPA (100*50 pixel sensor chip). The image was upscaled to $300 * 150$ pixel by pixel interpolation

\section{Summary}

Low-cost far-infrared sensor arrays were successfully fabricated using a high volume MEMS manufacturing process for pressure sensors. Advanced porous silicon membrane process (APSM) was used for fabrication of thermally isolated $\mathrm{Si}$ membranes. Thermodiodes were integrated in the membranes for temperature to voltage conversion. APSM provides monocrystalline membranes which is beneficial for reducing $1 / f$ noise of the thermodiodes.

Specification of the FPA was adapted to lowcost applications. For the $2^{\text {nd }}$ Gen FPA using $100 * 50$ pixels and $100 \mu \mathrm{m}$ pitch a thermal sensitivity (NETD) of $270 \mathrm{mK} @ \mathrm{f} / 1.0$ at $9 \mathrm{~Hz}$ frame rate was achieved. Future developments focus on further performance improvements for both $2^{\text {nd }}$ and $3^{\text {rd }}$ Gen FPA.

\section{Acknowledgements}

Part of the research leading to the results of $1^{\text {st }}$ and $2^{\text {nd }}$ Gen FPA has received funding from the European Community's Seventh Framework Programme under grant agreement $n^{\circ} 216049$, relating to the project "Reliable Application Specific Detection of Road Users with OnBoard Sensors (ADOSE)". Work on the $3^{\text {rd }}$ Gen FPA currently receives funding from the German ministry for education and research (BMBF) within the excellence cluster MicroTEC Südwest as project number 16SV5128 (RTFIR). 


\section{References}

[1] F. Niklaus, C. Vieider, H. Jakobsen, MEMS-

Based Uncooled Infrared Bolometer Arrays - A

Review, Proc. of SPIE Vol. 6836, 68360D,

(2007); doi: 10.1117/12.755128

[2] D. Takamuro et al., Development of new SOI diode structure for beyond $17 \mu \mathrm{m}$ pixel pitch SOI diode uncooled IRFPAs, Proc. SPIE, 8012, 80121E (2011); doi:10.1117/12.884556

[3] S. Armbruster et al, A novel micromachining process for the fabrication of monocrystalline Simembranes using porous silicon,

TRANSDUCERS, Solid-State Sensors, Actuators and Microsystems, 12th International Conference on, 2003 , vol.1, no., pp. 246- 249 vol.1, 8-12 June 2003 doi: 10.1109/SENSOR.2003.1215299

[4] D.L. Blackburn, Temperature measurements of semiconductor devices - a review, Proceedings of: Semiconductor thermal measurement and management symposium, 2004, Twentieth annual IEEE, p. 70-80, doi:

10.1109/STHERM.2004.1291304 